\title{
Enhanced Cost231 W.I. Propagation Model in Wireless Network
}

\author{
Hemant Kumar Sharma \\ School of IT, Rajiv Gandhi \\ Proudyogiki Vishwavidyalaya, \\ Bhopal (M.P) India
}

\author{
Santosh Sahu \\ School of IT, Rajiv Gandhi \\ Proudyogiki Vishwavidyalaya, \\ Bhopal (M.P) India
}

\author{
Sanjeev Sharma \\ School of IT, Rajiv Gandhi \\ Proudyogiki Vishwavidyalaya, \\ Bhopal (M.P) India
}

\begin{abstract}
Implementations of wireless network wave propagation models are necessary to determine propagation characteristic through a medium. Propagation study provides an estimation of signal characteristics. This paper evaluates about propagation models, their path loss behavior, propagation mechanisms, and propagation prediction techniques. A propagation model is a set of mathematical expressions, diagrams, and algorithms used to represent the radio characteristics of a given environment. The accuracy of any particular propagation model in any given condition will depend on the suitability among the constraints required by the model and depend on terrain. This paper proposes new semi deterministic model derived by walficsh and Ikagami, this model simulation results represents the accuracy in terms of through put, end to end delay, average jitter and total packet received with respect to cost 231 Hata model and cost 231 (W.I) models and also shows some limitations, proposed model is unable to calculate multiple reflection loss caused by multi screen.
\end{abstract}

\section{Keywords}

Propagation mechanism, Propagation prediction techniques, propagation models.

\section{INTRODUCTION}

Commercial success of wireless, since its initial implementation has lad to an interest between wireless engineers in understanding and predicting radio propagation characteristic in different urban and suburban area, expansive growth of wireless network, it is very precious to have the capability of determining path loss.

The development of efficient transmission, operation and management technologies and a progressive reduction in the size of the cells requires a greater suitability on the estimations of the system coverage, which is given by propagation losses, in order to obtain "total coverage" with which the operator attempts to assurance the quality of service. For this reason an accurate and flexible prediction methodologies of coverage with easy implementation is required [1]. It is hard to find a methodology of signal prediction which achieves the precision-complexitytime paradigm. The aim of finding a single methodology for any metropolitan environment is insufficient because it is clear that the performance of a system is closely related to the operation region. The main aim of this paper to find the accurate propagation model for the radio channel attenuation for metropolitan area, Wave propagation models are necessary to determine propagation characteristics for any random mechanism. An understanding of radio propagation [2] is essential for coming up with appropriate design, deployment, and management strategies for any wireless network. In the next section brief overview of propagation mechanism, section III. discuss main propagation model, section IV is describing propose work, section $\mathrm{V}$ is Computer Simulation and used methodology and section VI is results analysis and last section is conclusion.

\section{PROPAGATION MECHANISM}

This section discusses main propagation mechanism, reflection, single diffraction, multiple diffraction, scattering, penetration, absorption. Reflection [3][4] occurs when a propagating electromagnetic wave impinges upon an object that has very large dimensions compared to the wavelength of the propagating wave. Reflection occurs from the surface of the ground, from walls, and from furniture. Diffraction [5] According to Huygen's principle, all points on a wave front are point sources of secondary waves propagating to all directions. Therefore, each time a radio wave passes an edge such as a corner of a building the wave "bends" around the edge and continues to propagate into the area shadowed by the edge. This effect is called diffraction. Scattering [6] phenomena occurs when the medium through which the wave travels is composed of objects with small dimensions, when compared to the wavelength, and where the number of obstacles is large. Scattered waves are produced when waves impinge on rough surfaces, foliage and small objects in general. Absorption is a loss that occurs if the signal passes through varying mediums or obstacles in which some of the transmitted signal is converted into another form of energy, usually thermal, and some of it continues to propagate

All propagation mechanism is shown in figure1.

A. For specular reflection the field is proportional to $1 /$ $\left(d_{1}+d_{2}\right)$.

B. For single diffraction, the field is proportional to $\left(\mathrm{d}_{1} / \mathrm{d}_{2}\right.$ $\left.\left(d_{1}+d_{2}\right)\right)^{-0.5}$.

C. For multiple diffraction and for a source illuminating all edges, the field is proportional to $1 / \mathrm{d}^{1.9}$.

D. For volume scattering and rough surface scattering, the field is proportional to $1 /\left(\mathrm{d}_{1} \cdot \mathrm{d}_{2}\right)$.

E. For penetration and absorption, the field is mainly attenuated by a constant.

F. For the wave guiding phenomena, the logarithm of the field is proportional to $\mathrm{d}$. 


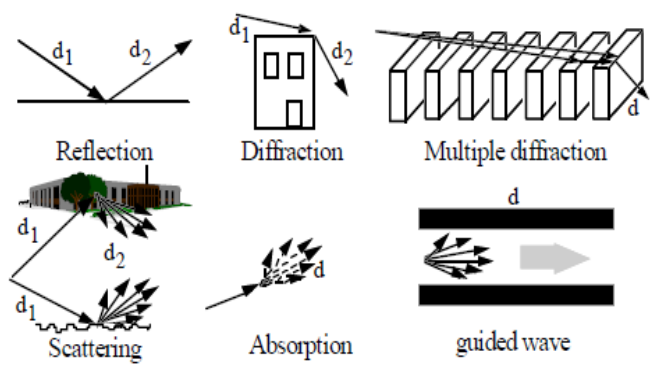

Fig.1. Propagation phenomena

\section{PROPAGATION MODELS}

Propagation models [7] in wireless communication have focused on predicting the average received signal strength at a given distance from the transmitter as well as the inconsistency of the signal strength in close proximity to a particular location. Propagation models that predict the mean signal strength for an random transmitter - receiver separation distance are useful in estimating the radio Coverage area of transmitter and are called large scale propagation models. The propagation models are generally used to differentiate the quality of mobile communication. A propagation model is a set of mathematical expressions, diagrams, and algorithms used to represent the radio characteristics of a given environment. Propagation model are three types empirical model, semi deterministic model, deterministic model Empirical models are based on measurement data, simple, use statistical properties,



Fig. 2. Block diagram for propagation loss calculations

And not very accurate Semi-deterministic models are based on empirical models and deterministic aspects. Deterministic models are site-specific, require enormous number of geometry information about the cite, very important computational effort, accurate. In Figure 2 we are showing basic steps of path loss calculation [9]. Figure show what are the important factor for calculating path loss in wireless network.

\subsection{Free Space Model}

This is a large scale model. The received power is only dependent on the transmitted power, the antenna's gains and on the distance between the sender and the receiver. It accounts mainly for the fact that a radio wave which moves away from the sender has to cover a larger area. So the received power decreases with the square of the distance. The free space propagation model assumes the ideal propagation condition that there is only one clear line-of-sight path between the transmitter and receiver. H. T. Friis presented the following equation to calculate the received signal power in free space at distance $d$ from the transmitter [8] [16].

$$
P_{R}=P_{T} \cdot G_{T} \cdot G_{R \cdot}\left(\frac{\lambda}{4 \pi d}\right)^{2}
$$

Where $P_{T}$ is the transmitted signal power $G_{T}$ and $G_{R}$ are the antenna gains of the transmitter and the receiver respectively. $\lambda$ is the wavelength. It is common to select $G_{T}=G_{R}=1$.then,

$$
\frac{P_{T}}{P_{R}}=\left(\frac{4 \pi d}{\lambda}\right)^{2}=\left(\frac{4 \pi d f}{c}\right)^{2}
$$

Expressed in $\mathrm{dB}$ as:

$$
\begin{array}{r}
L(d B)=10 \log \frac{P_{T}}{P_{R}}=10 \log \left(\frac{4 \pi d f}{c}\right)^{2}=20 \log \left(\frac{4 \pi d f}{c}\right) \\
L(d B)=32.44+20 \log (d k m)+20 \log (F M h z)
\end{array}
$$

One is able to see from the above free space equations that $6 \mathrm{~dB}$ of loss is associated with a doubling of the frequency. This same relationship also holds for the distance, if the distance is doubled, $6 \mathrm{~dB}$ of additional loss will be encountered.

\subsection{Two Ray Ground Model}

One more popular path loss model is the two-ray model or the two-path model. The free space model described above assumes that there is only one single path from the transmitter to the receiver. But in reality the signal reaches the receiver through multiple paths (because of reflection, refraction and scattering). The two-path model tries to capture this phenomenon. The model assumes that the signal reaches the receiver through two paths, one a line-of-sight path, and the other the path through which the reflected wave is received. According to the two-path model, the received power is given by [10],

$$
P_{r}=P_{t} \cdot G_{t} \cdot G_{r}\left(\frac{h_{t} \cdot h_{r}}{d^{2}}\right)^{2}
$$

Where Pt is the transmitted power, Gt and Gr are the transmitter and receiver antenna gains, respectively, in the direction from the transmitter and receiver, $d$ is the distance between the transmitter and receiver, and ht and hr are the heights of the transmitter and receiver, respectively.

\subsection{Okumura Model}

This section discusses different propagation techniques Okumara is the first technique to find path loss in urban area. Okumura's model [11] [15] is most widely used for signal predictions in urban and suburban areas operating in the range of 
$150 \mathrm{MHz}$ to $1.92 \mathrm{GHz}$. The basis of the method is that the free space path loss between the transmitter and receiver is added to the value of $A_{m u}(f, d)$, where $A_{m u}$ is the median attenuation, relative to free space in an urban area with a base station effective antenna height $\mathrm{H}_{\mathrm{te}} 200$ meter and mobile station height $\mathrm{H}_{\mathrm{re}}$ is 3 meter To determine path loss using Okumura's model, the free space path loss between the points of interest is first determined, and then the value of $\mathrm{A}_{\mathrm{mu}}$ ( $\mathrm{f}, \mathrm{d}$ ) is added to it along with correction factors to account for the type of terrain as expressed in.

$$
L_{50}(d B)=L_{f}+A_{m u}-G_{H t e}-G_{G H r e}-G_{\text {area }}
$$

Here, $\mathrm{L}_{50}(\mathrm{~dB})$ is the 50 percentile value of propagation path loss, $\mathrm{L}_{\mathrm{F}}$ is propagation loss of free space, $\mathrm{A}_{\mathrm{mu}}$ is the median attenuation relative to free space, $\mathrm{G}_{\mathrm{Hte}}, \mathrm{G}_{\mathrm{Hre}}, \mathrm{G}_{\text {area }}$ are BTS antenna height gain factor, mobile antenna height gain factor and gain due to the type of environment. Okumara model is empirical in nature means that all parameters are used to specific range determined by the measured data. If the user finds that one or more parameters are outside the range then there is no alternate.

\subsection{Hata Propagation Model}

The Hata model is an empirical formulation [12] of the graphical path-loss data provided by Okumura's model. The formula for the median path loss in urban areas is given by

$$
\begin{aligned}
L_{b}(\text { urban })(d B)= & 69.55+26.16 \log f c-13.82 \log \text { hte } \\
& -a(\text { hre })+(44.9-6.55 \log \text { hte }) . \log d
\end{aligned}
$$

Where $f_{c}$ is the frequency (in $\mathrm{MHz}$ ), which varies from 150 $\mathrm{MHz}$ to $1500 \mathrm{MHz} \mathrm{h}_{\mathrm{te}}$ and $\mathrm{h}_{\mathrm{re}}$ are the effective heights of the base station and the mobile antennas (in meters), respectively. $\mathrm{d}$ is the distance from the base station to the mobile antenna, $\mathrm{a}\left(\mathrm{h}_{\mathrm{re}}\right)$ is the correction factor for the effective antenna height of the mobile unit, which is a function of the size of the area of coverage. For small- to medium-sized cities, the mobile-antenna correction factor is given by:

$$
a\left(h_{r e}=\left(1.1 \log f_{c}-0.7\right) h_{r e}-\left(1.56 \log f_{c}-0.8\right) d B\right.
$$

path loss in suburban area

$$
L_{b}(d B)=L_{b}(\text { urban })-2\left[\log \left(\frac{f_{c}}{28}\right)\right]^{2}-5.4
$$

The path loss in open rural area as is expressed

$$
L_{b}(d B)=L_{b}(u r b a n)-4.78\left(\log f_{c}\right)^{2}-18.33 \log f_{c}-40.98
$$

These equation improved performance value of okumara model, this technique is good in urban and suburban area but in rural areas performance degreases because rural area prediction is depend on urban area. This model is quite suitable for large-cell mobile systems, but not for personal communications systems that cover a circular area of approximately $1 \mathrm{~km}$ in radius.

\subsection{Walfisch and Bertoni model}

A model developed by Walfisch and Bertoni [13] considers the impact of rooftops and building heights by using diffraction to predict average signal strength at street level. It is a semi- deterministic model. The model considers the path loss, S, to be the product of three factors:

$$
S=P_{0} \cdot Q^{2} \cdot P_{1}
$$

Where $\mathrm{P}_{0}$ is the free space path loss between isotropic antennas given by:

$$
P_{0}=(\lambda / 4 \pi R)^{2}
$$

The factor $\mathrm{Q}^{2}$ reflects the signal power reduction due to buildings that block the receiver at street level. The $\mathrm{P}_{1}$ term is based on diffraction and determines the signal loss from the rooftop to the street. The model has been adopted for the IMT2000 standard.

\subsection{Cost 231(Walfisch and Ikegami) Model}

This empirical model is a combination of the models from $\mathrm{J}$. Walfisch and F. Ikegami. It was developed by the COST 231 project. It is now called Empirical COST-Walfisch-Ikegami Model. The frequency ranges from $800 \mathrm{MHz}$ to $2000 \mathrm{MHz}$. This model is used primarily in Europe for the GSM1900 system [14] [15]

Path Loss,

$$
L_{50}(d B)=L_{f}+L_{r t s}+L_{m s d}
$$

Where

$\mathrm{L}_{\mathrm{f}}=$ free-space loss

$\mathrm{L}_{\mathrm{rts}}=$ rooftop-to-street diffraction and scatter loss

$\mathrm{L}_{\mathrm{msd}}=$ multi screen loss

Free space loss is given as

$$
L_{f}=32.4+20 \log d+20 \log f_{c} d B
$$

The rooftop-to-street diffraction and scatter loss is given as [14]

$$
\begin{aligned}
& L_{\text {rts }}=-16.9-10 \log \left(\frac{w}{m}\right)+ \\
& 10 \log \left(\frac{f}{M H z}\right)+20 \log \left(\frac{\Delta h_{\text {mobile }}}{m}\right)+L_{\text {Ori }} \\
& L_{\text {Ori }}= \\
& \qquad \begin{array}{l}
-10+0.354 \frac{\varphi}{d e g} \text { for } 0^{\circ} \leq \varphi<35^{\circ} \\
2.5+0.075\left(\frac{\varphi}{d e g}-35\right) \text { for } 35^{0} \leq \varphi<55^{\circ} \\
4.0+0.114\left(\frac{\varphi}{\text { deg }}-55\right) \text { for } 55^{0} \leq \varphi<90^{\circ}
\end{array}
\end{aligned}
$$

With $w=$ width of the roads

Where $\mathrm{L}_{\text {ori }}=$ Orientation Loss

$\varphi=$ incident angle relative to the street 
The multi screen loss is given as:

$$
L_{m s d}=L_{b s h}+k_{a}+k_{d} \log d+k_{f} \log f_{c}-9 \log b
$$

This model is restricted to the following range of parameter: frequency range of this model is 800 to $2000 \mathrm{MHz}$ and the base station height is 4 to $50 \mathrm{~m}$ and mobile station height is 1 to $3 \mathrm{~m}$, and distance between base station and mobile station $\mathrm{d}$ is 0.02 to $5 \mathrm{~km}$.

\section{PROPOSED WORK}

This paper proposed new cost 231 (walfisch and Ikagami) model. Cost 231 wi model has limitations, this model calculate only rooftop-to-street diffraction and scatter loss and multi screen diffraction loss. It cannot calculate loss due to multiple reflections. In extended cost wi model we add the new term $\mathrm{L}_{\mathrm{sv}}$ which is used to calculate multiple reflection within building block. The model gives a propagation loss function $\mathrm{L}$ with the following parameters: frequency $f$ base station height $h_{\text {base }}$, MS height $\mathrm{h}_{\mathrm{m}}$ distance between the MS and the BS d, street width $w$, angle between the street and propagation direction $\varphi$, building height $\mathrm{h}_{\text {roof }}$, building separation $b$, and terrain height.

New model is:

$$
L_{50}(d B)=L_{f}+L_{r t s}+L_{m s d}+L_{s v}
$$

Where

Path loss in Line of sight condition

$$
L_{f}=42.6+26 \log d+20 \log f_{c} d B \text { for } d>20
$$

Path loss in NLOS-condition

The basic transmission loss is composed of the terms free space loss $L_{0}$, multiple screen diffraction loss $L_{m s d}$, and roof-top-tostreet diffraction and scatters loss $L_{r t s}$ and signal variation loss Lsv.

$$
L_{f}=32.4+20 \log d+20 \log f_{c} d B
$$

Roof-top-to-street diffraction and scatters loss:

$L_{r t s}=-16.9-10 \log \left(\frac{w}{m}\right)+$

$10 \log \left(\frac{f}{M H z}\right)+20 \log \left(\frac{\Delta h_{\text {mobile }}}{m}\right)+L_{\text {Ori }}$

And multi screen loss $\mathrm{L}_{\mathrm{msd}}$

$$
L_{m s d}=L_{b s h}+k_{a}+k_{d} \log \left(\frac{d}{k m}\right)+
$$

$k_{f} \log \left(\frac{f}{M H z}\right)-9 \log \left(\frac{b}{m}\right)$

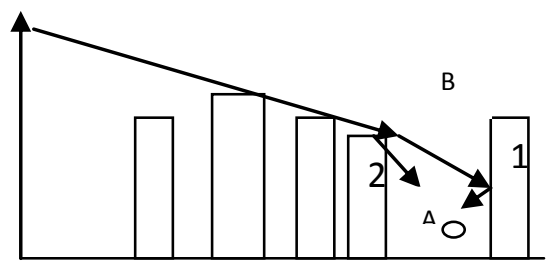

Fig.3. Two rays at the MS considered by models

New Model considers two main rays arriving to the mobile as shown in Figure 3. The first one is the ray that, is diffracted by the least building (1) and arrives directly to the mobile. The second ray is diffracted by building (1) and reflected in the building (2).This is new extended cost 321 wi model. The term $\mathrm{L}_{\mathrm{f}}$ and $\mathrm{L}_{\mathrm{rts}}$ are same as old model. In $\mathrm{L}_{\mathrm{msd}}$ We change the value of $\mathrm{k}_{\mathrm{d}}$.

$$
L_{s v}=-11.32+3.3\left[\log \left(d_{s v 1}\right)+\log \left(d_{s v 2}\right)\right]
$$

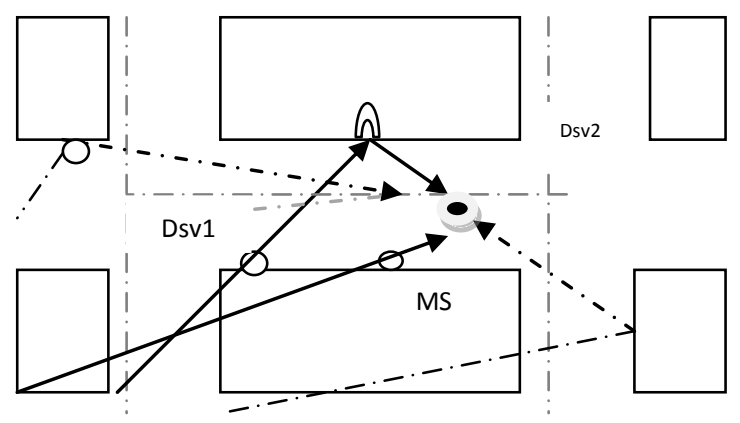

\section{Fig. 4 Rays to be considered in the case of finite screens} reaching the MS coming from the corners

New model add a loss term $\mathrm{L}_{\mathrm{sv}}$ with a logarithmic dependence with the distances from each street intersection, dsv1 and $d_{\mathrm{sv} 2}$ has empirically been adjusted based on field measurements. Additional rays should be accounted as main rays as they follow the criterion of diffraction or a diffraction-reflection. Additional rays suffer a diffraction on the edges placed at the corners and diffraction-reflection which occurs in the crossing of the streets.

\section{COMPUTER SIMULATION}

In this paper all the simulation work is performed in QualNet [17] wireless network simulator version 5.0. Initially number of nodes are 10, Simulation time was taken 200 seconds and seed as 1 . All the scenarios have been designed in $500 \mathrm{~m} \times 500 \mathrm{~m}$ area. Mobility model used is Random Way Point (RWP). In this model a mobile node is initially placed in a random location in the simulation area, and then moved in a randomly chosen direction between at a constant speed. The movement proceeds for a specific amount of time or distance, and the process is repeated a predetermined number of times. Node in network moves with constant speed of $10 \mathrm{~m} / \mathrm{s}$ and with pause time of $0 \mathrm{~s}$ to 50s. All the simulation work was carried out using cost 231 Hata model, cost 231 wi and its variant with AODV routing protocol .Network traffic is provided by using constant Bit Rate (CBR) application. 


\section{Simulation Methodology}

\begin{tabular}{|l|l|l|l|l|l|l|}
\hline $\begin{array}{l}\text { Simulat } \\
\text { on Time }\end{array}$ & Node & Area & $\begin{array}{l}\text { Pause } \\
\text { Time }\end{array}$ & $\begin{array}{l}\text { Propag } \\
\text { ation } \\
\text { Model }\end{array}$ & $\begin{array}{l}\text { Routin } \\
\text { g } \\
\text { Protoc } \\
\text { ol }\end{array}$ & $\begin{array}{l}\text { Noded } \\
\text { (m/sec) }\end{array}$ \\
\hline Const. & Const. & Const & $\begin{array}{l}\text { Will } \\
\text { Change }\end{array}$ & $\begin{array}{l}\text { Will } \\
\text { Change }\end{array}$ & Const. & Const. \\
\hline Const. & $\begin{array}{l}\text { Will } \\
\text { Change }\end{array}$ & Const & Const. & $\begin{array}{l}\text { Will } \\
\text { Change }\end{array}$ & Const. & Const. \\
\hline
\end{tabular}

Performance metrics used for this works are as follows:

Throughput is the measure of the number of packets successfully transmitted to their final destination per unit time. It is the ratio between the numbers of sent packets vs. received packets.

Average End to End Delay signifies the average time taken by packets to reach one end to another end (Source to Destination).

Average Jitter Effect signifies the Packets from the source will reach the destination with different delays. A packet's delay varies with its position in the queues of the routers along the path between source and destination and this position can vary unpredictably.

Total Bytes received are the measure of total packet received by server. The packets may be drop due to heavy traffic. So received packets may be vary according to traffic conditions.

\section{RESULTS AND ANALYSIS}

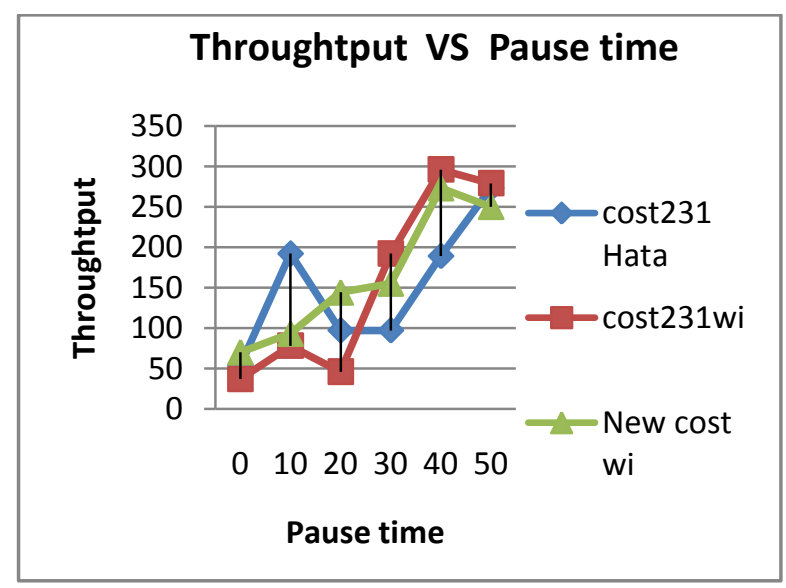

Fig. 5

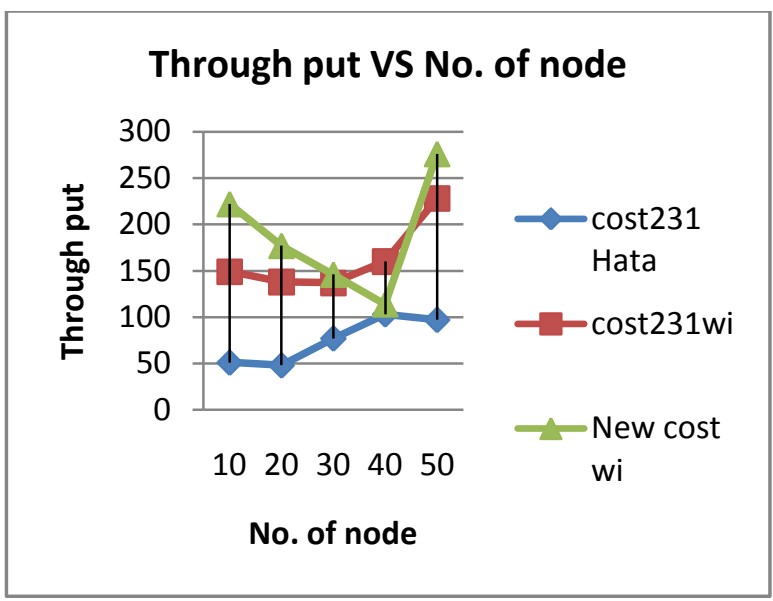

Figure 6

Figure 5 \& 6 shows throughput of different propagation models variation in pause time and number of nodes. Throughput is the ratio of numbers of sent packets with number of received packets. It has to be seen that Throughput of new cost wi is better than other model when pause time zero and number of node are minimum.



Figure 7

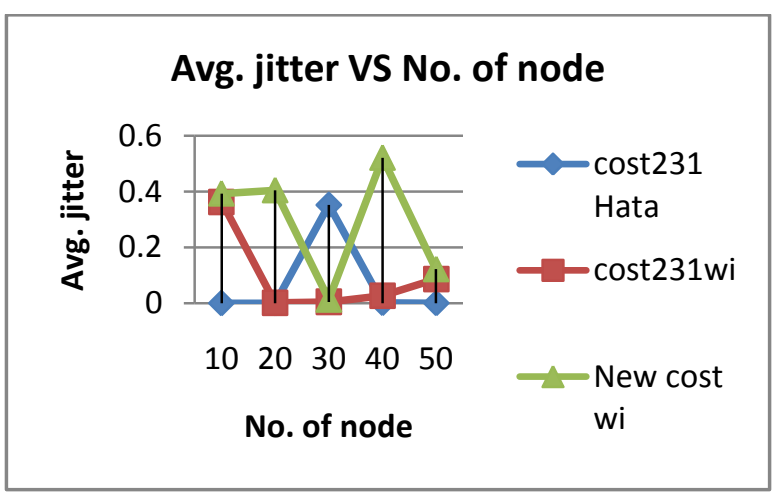

Figure 8

Figure $7 \& 8$ shows Average jitter of different propagation models, variation in pause time and number of nodes. Average Jitter Effect signifies the Packets from the source will reach the destination with different delays. Average jitter of new model is higher than other models. 


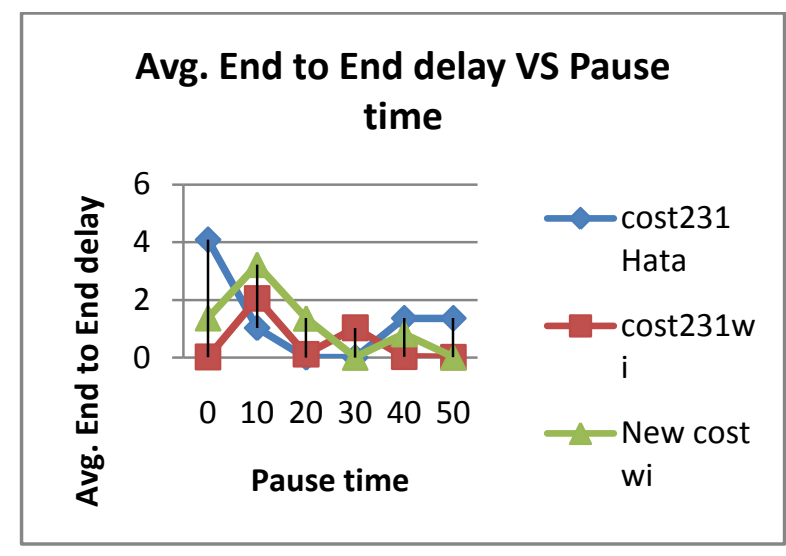

Figure 9

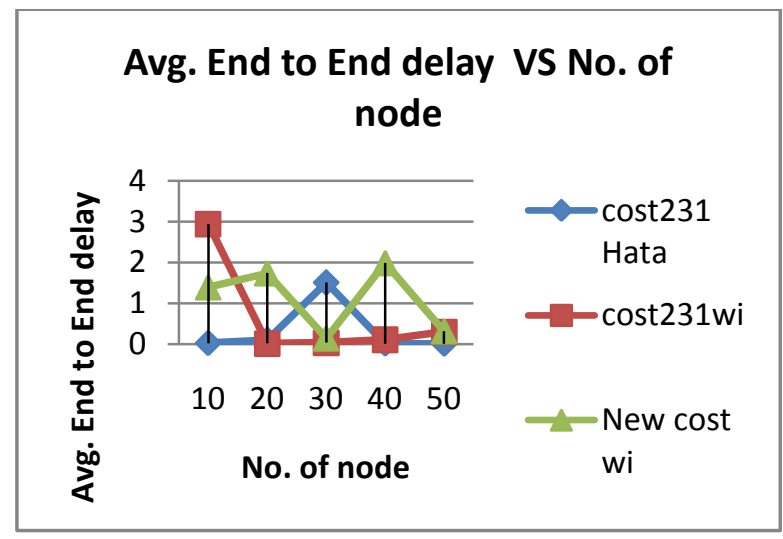

Figure 10

Figure $9 \& 10$ shows Average end to end delay of different propagation models variation in pause time and number of nodes. End to end delay is not good in new cost 231 wi model.

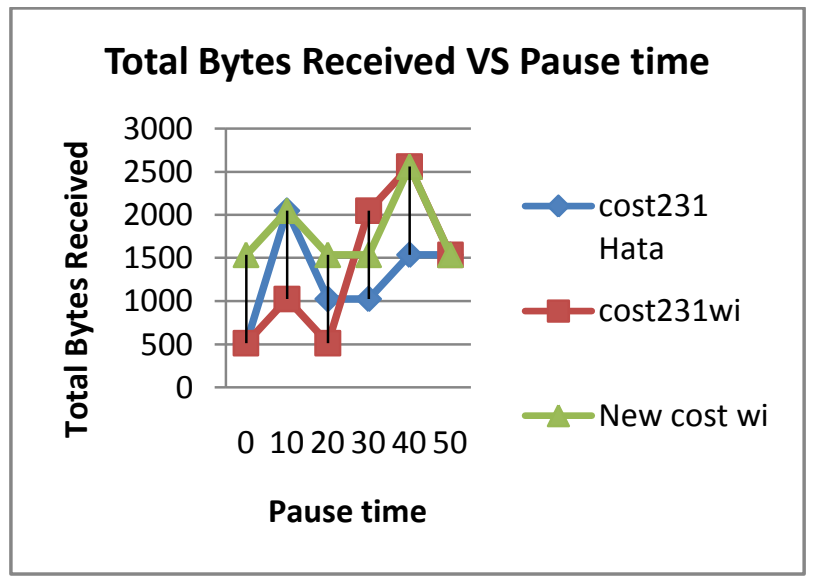

Figure 11

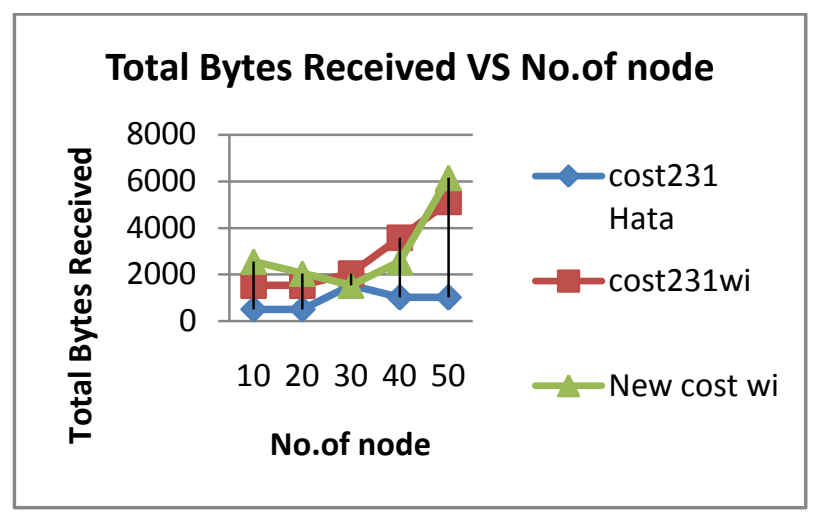

Figure 12

Figure $11 \& 12$ shows the readings of total byte received for different propagation model with variation in pause time and number of node in network. It has to be seen that receiver can receive maximum byte at new cost wi, but it is sometimes less due to congestion in network.

\section{CONCLUSION}

This paper discusses cost 231 Hata model and Cost 231 walficsh Ikagami model, but both models are not efficient and accurate in urban environment, there are some limitations in these model, such that multiple reflection due to multiple screen in urban environment. Proposed model removes these limitations and improve throughput in comparison to the cost 231 hata and cost 231 walficsh Ikagami, all simulation work is done in Qual Net5.0. This paper compare new cost 231 wi model with respect to cost 231 hata and cost 231 walficsh Ikagami model, performance metrics used for this work is Throughput, Average End to End Delay, Average Jitter, Total Bytes received. Through put of new model is superior when increasing number of node as well as increasing pause time, but average jitter and average end to end delay is high. New cost 231 wi model improve performance and accuracy. The main aim of this research is to find a propagation model for the radio channel attenuation in the frequency range $1800 \mathrm{MHz}$ in urban environment. This model has been developed for urban areas of small macro cells.

\section{REFERENCES}

[1] Casaravilla J., Dutra G., Pignataro N. \& Acuna J. "Propagation Model for Small Macro cells in Urban Areas" IEEE transactions on vehicular technology, vol. 58, no. 7, september 2009.

[2] H. L. Bertoni, "Radio Propagation for Modern Wireless Systems" Upper Saddle River, NJ, Prentice Hall PTR, 2000, pp. 90-92.

[3] Thomas Kurner and Dieter J. Cichon "Propagation Prediction Models".

[4] Mark r. alexander "understanding and predicting urban propagation losses" naval postgraduate school monterey, california September 2009.

[5] H. Sizun, P.de Fornel, "Radio Wave Propagation for Telecommunication Applications" Springer, ISBN: 978$3540407584,2004$. 
[6] F.D.Cardoso, S.Taccori, L.M.Correia, IST-2001-32125 FLOWS, “Initial Propagation Models". pp.20, 2002.

[7] Nagendra sah and Amit Kumar "CSP Algorithm in Predicting and Optimizing the Path Loss of Wireless Empirical Propagation Models"International Journal of Computer and Electrical Engineering, Vol. 1, No. 4, October, 2009.

[8] James Demetriou and Rebecca MacKenzie "Propagation Basics" September 30, 1998.

[9] Robert K. Crane "Propagation Handbook for Wireless Communication System Design".

[10] Ayyaswamy Kathirvel, and Rengaramanujam Srinivasan "Analysis of Propagation Model using Mobile Ad Hoc Network Routing Protocols" International Journal of Research and Reviews in Computer Science (IJRRCS), Vol. 1, No. 1.

[11] Sagar Patel, Ved Vyas Dwivedi and Y.P. Kosta "A Parametric Characterization and Comparative Study of Okumura and Hata Propagation-loss prediction Models for Wireless Environment" Number 4 (2010).
[12] Tapan K. Sarkar, Zhong Ji, Kyungjung Kim, Abdellatif Medour "A Survey of Various Propagation Models for Mobile Communication" IEEE Antennas and Propagation Magazine, Vol. 45, No. 3, June 2003.

[13] Theodore S. Rappaport, 2002, Wireless Communications: Principles and Practice, Prentice Hall PTR.

[14] R. Mardeni and K. F. Kwan "optimization of hata propagation prediction model in suburban area in Malaysia" Progress In Electromagnetics Research C, Vol. 13, 106, 2010.

[15] J.d. parsons "The mobile radio propagation channel" second edition, Jon welly and sons Ltd.

[16] Ibrahim Khider Eltahir "The Impact of Different Radio Propagation Models for Mobile Ad hoc NETworks (MANET) in Urban Area Environment" The 2nd International Conference on Wireless Broadband and Ultra Wideband Communications (Aus Wireless 2007).

[17] Scalable Network Technology, "QualNet5.0 simulator" tutorial and QualNet Forum. 